\title{
Enfoque de Manejo Costero Integrado aplicado a la cuenca baja del arroyo El Potrero, Maldonado, Uruguay
}

\section{The Integrated Coastal Management approach in the lower basin of the El Potrero stream, Maldonado, Uruguay}

Bergós, Lucía (1); Cardozo, Juan Manuel (1); Bruzzone, Carla (1).

(1) Maestría en Manejo Costero Integrado del Cono Sur, Universidad de la República (UdelaR).

Contacto: juancebal@gmail.com

RECIBIDO: 1/4/2017 - APROBADO: 30/6/2017

\begin{abstract}
Resumen
En la cuenca del arroyo El Potrero, departamento de Maldonado, confluyen una particular diversidad de ambientes, actores, intereses y problemáticas socioambientales. El objetivo de este trabajo es proponer medidas para el manejo de los conflictos, problemáticas y oportunidades socioambientales en el área, aplicando herramientas de Manejo Costero Integrado (MCI). El MCI busca un balance entre intereses sociales, ambientales y económicos en la zona costera, con un enfoque basado en ecosistemas e incorporando en el proceso a los distintos actores de una comunidad dada. A partir de revisión documental, entrevistas a 11 actores con incidencia local, talleres internos del equipo de trabajo y un taller abierto de validación se reconocieron cuatro asuntos sobre los que enfocar las propuestas de manejo: fortalecimiento de la gobernanza, fomento de la integridad ambiental de la costa balnearia, conservación del arroyo y sus márgenes y consolidación de una trama urbana de bajo impacto ambiental. Cada asunto de manejo dio origen a un programa del cual se desprenden proyectos con acciones específicas. Todos los programas se agrupan en un «Plan integrado de manejo de la cuenca baja del arroyo El Potrero». El espacio de intercambio entre actores diversos promovido en este estudio favoreció la generación de nuevos lazos y mostró el potencial del trabajo conjunto.

Palabras clave: Enfoque integral, interdisciplina, plan de manejo.
\end{abstract}

\begin{abstract}
A diversity of environments, stakeholders, interests and socioenvironmental problems converge in the basin of the El Potrero stream, Maldonado department, Uruguay. The objective of this article is to outline management proposals for conflicts, problems and socioenvironmental opportunities in this area, applying the Integrated Coastal Management (ICM) approach. The ICM promotes a balance between social, environmental, and economic interests in the coastal zone, based on an ecosystem approach and involving different community stakeholders in the process. Based on document reviews, interviews with 11 local actors, internal team workshops, and an open validation workshop, four issues were identified to focus the management proposals: strengthening governance, promoting the environmental integrity of the seaside coast, conserving the stream and its margins, and fostering urbanization with a low environmental impact. Each management issue gave rise to a program from which projects with specific actions emerged. All programs are grouped in an «Integrated Management Plan for the Lower Basin of the El Potrero stream». The exchange between different actors favored the relationship between stakeholders and showed the potential for working together.

Keywords: Integral approach, Interdiscipline, Management plan.
\end{abstract}

\section{Introducción}

El Manejo Costero Integrado (MCI) es una estrategia de gestión que ha adquirido especial relevancia a partir de la Agenda 21, producto de la Cumbre de la Tierra celebrada en Río de Janeiro en 1992, donde se plasmó la necesidad de una «ordenación integrada y desarrollo sostenible de las zonas costeras y las zonas marinas» (United Nations, 1993, Capítulo
17, apartado 17.1.a). El MCI es «un proceso participativo que busca integrar los diversos niveles gubernamentales, la comunidad, la ciencia y los intereses sectoriales y públicos, en la elaboración e implementación de programas para la protección y el desarrollo sustentable de los recursos y ambientes costeros» (Hildebrand, 2002; en Conde, 2013, p. 30). Busca alcanzar un equilibrio entre los usos de la costa y mejorar la calidad de vida de las comunidades y grupos humanos que dependen 
de los recursos costeros, manteniendo a su vez la diversidad y productividad biológica de esos ecosistemas (GESAMP, 1999). Concibe, por tanto, a la zona costera, con sus procesos y actores, como un sistema socioecológico (Ostrom, 2009). Sus límites de acción incluyen la costa y tierras aledañas, desde las cuencas hidrográficas altas hasta los límites exteriores de las Zonas Económicas Exclusivas, con una visión de continuo tierra-costa-océano. Este enfoque propone un proceso de planificación interactivo y adaptativo, en forma de ciclos consecutivos usualmente de cinco etapas: 1) identificación de asuntos clave de manejo, que supone principalmente la identificación de los principales asuntos ambientales, sociales e institucionales y la identificación de actores y sus intereses; 2) preparación del programa, entendido como la preparación del plan de manejo; 3) adopción formal, que implica obtener el respaldo gubernamental y desarrollar el marco institucional necesario y el financiamiento; 4) implementación propiamente dicha, y 5) evaluación (Ochoa y Olsen, 2007).

La perspectiva del MCI ha sido implementada para el manejo de zonas costeras en más de 100 países. Si bien no hay datos recientes, se ha constatado un aumento de las iniciativas a nivel mundial, de 217 experiencias en 1993 a aproximadamente 700 en el año 2000 (Sorensen, 2002). En particular en Uruguay el MCI ha sido promovido en espacios de gestión interinstitucional desde el año 1999 (Aguirre, et al., 2001) y desde espacios académicos a partir del año 2005 (CIMCI, 2011). En las cuatro ediciones que han transcurrido de la maestría en Manejo Costero Integrado del Cono Sur de la Universidad de la República se han desarrollado experiencias como la que aquí se presenta, donde se avanzó en el desarrollo de propuestas de manejo para diferentes tramos de las costas del Río de la Plata y Océano Atlántico en Uruguay (CIMCI, 2011). En ninguno de los casos, así como tampoco en el que se presenta en este trabajo, se avanzó en la implementación de las medidas propuestas. Diversas instituciones a nivel del gobierno nacional y departamental desarrollan prácticas que tienden a un MCI, aunque en la mayoría de los casos la perspectiva es exclusivamente disciplinar (Baliero, et al., 2006).

En este trabajo se aplicó el enfoque MCI en la cuenca baja del arroyo El Potrero, departamento de Maldonado, Uruguay, para la generación de propuestas de manejo que atendieran a los asuntos más relevantes para el área, priorizando la perspectiva de los actores con incidencia local.

La cuenca del arroyo El Potrero se caracteriza por la confluencia de unidades ambientales con biodiversidad y usos antrópicos diferentes pero interconectados: la Laguna del Sauce, el arroyo El Potrero, a través del cual se produce la descarga de la laguna, y la playa, donde desemboca el arroyo en el Río de la Plata (ITU-IDM, 2009). Ocurren a su vez urbanizaciones de tipo balneario costero y predios rurales sobre la margen este del arroyo, que presentan una gran complejidad a nivel socio-territorial y ambiental. Los procesos y actividades humanas interaccionan con la matriz biofísica del sistema, generando conflictos socioambientales que deben ser atendidos desde un enfoque integral de manejo y gestión.

El objetivo de este trabajo es proponer medidas para el manejo de los conflictos, problemáticas y oportunidades socioambientales en la cuenca baja del arroyo El Potrero, aplicando herramientas de MCI. En este trabajo se muestran los resultados obtenidos en el desarrollo de las dos primeras

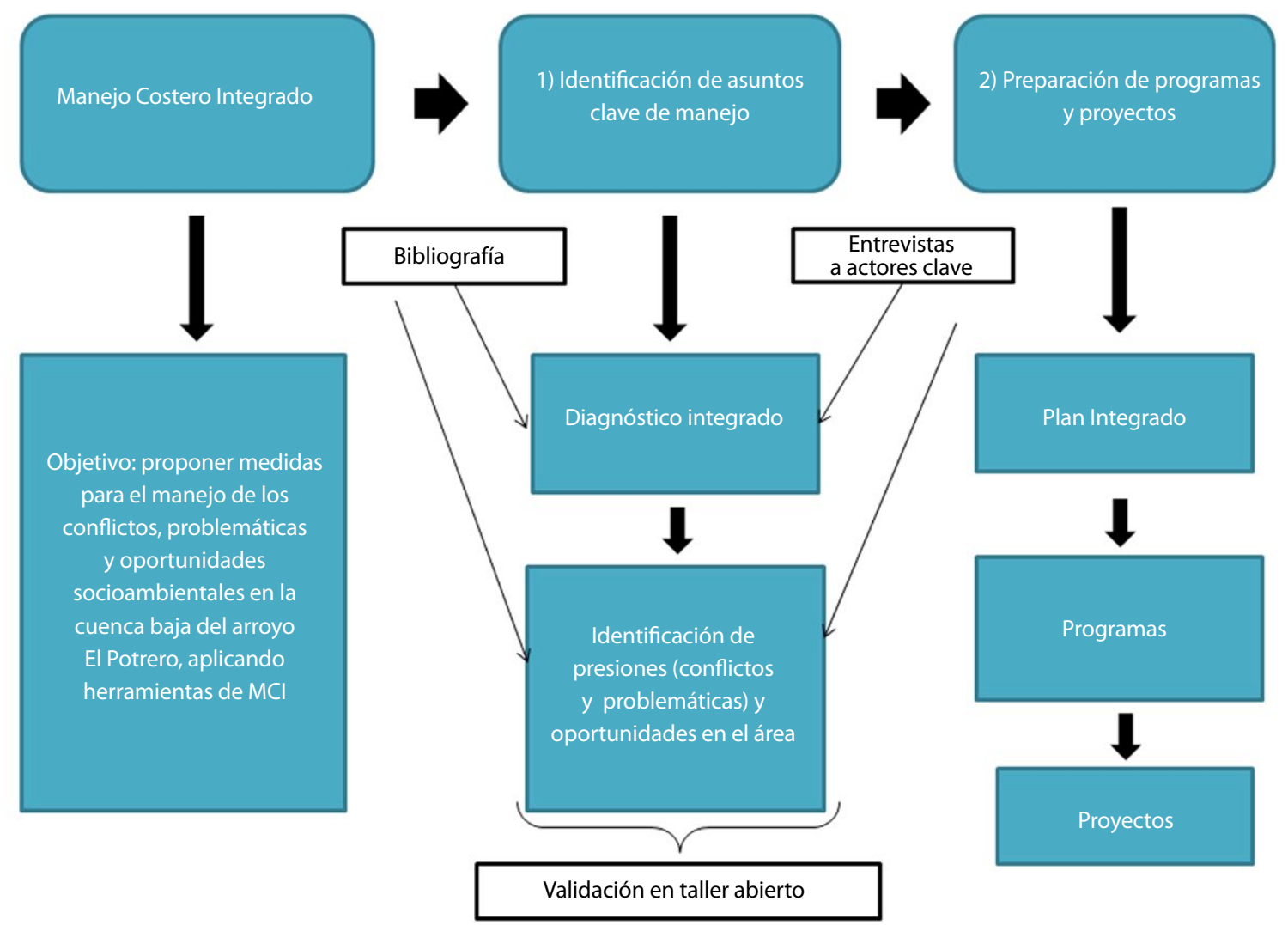

Figura 1. Estrategia general del trabajo y principales elementos considerados para su desarrollo. Fuente: elaboración propia. 
etapas del ciclo de MCI: la identificación de los asuntos clave de manejo para la zona de estudio y la preparación de programas y proyectos que atiendan los principales conflictos socioambientales (Figura 1). Además, se reflexiona en torno al valor del proceso de MCI en sí mismo como promotor de transformaciones en temáticas socioambientales.

\section{Materiales y Métodos}

\section{Área de estudio}

El área de estudio de este trabajo se encuentra circunscripta a la cuenca baja del arroyo El Potrero, ubicada al suroeste del departamento de Maldonado en Uruguay ( $34^{\circ} 52^{\prime} 31^{\prime \prime}$ S, 5506'25” W) (Figura 2). Comprende los balnearios costeros Sauce de Portezuelo, Ocean Park y Chihuahua, así como los predios rurales y suburbanos linderos al curso del arroyo hacia el sur de la ruta Interbalnearia No93, contenidos en un arco de playa de $20 \mathrm{~km}$ de extensión delimitado por las puntas rocosas de Punta Rasa al oeste y Punta Ballena al este.

\section{Estrategia metodológica}

\section{1) Identificación de asuntos clave de manejo: diagnóstico integrado}

La identificación de asuntos clave de manejo, primera etapa del ciclo de MCI, implicó previamente la realización de un diagnóstico integrado del área.

La elaboración de una propuesta de MCI implica determinar asuntos clave de manejo, entendidos como «problemas u oportunidades sobre los cuales se enfoca una iniciativa para introducir cambios en su curso y en sus resultados» (Olsen y Ochoa, 2004). La selección de los asuntos de manejo se basó, por tanto, en los conflictos, problemáticas y oportunidades reconocidos a partir de la información y opinión de actores clave vinculados al área, relevadas durante la etapa de diagnóstico y procesada en talleres internos del grupo de trabajo. Se entendió como actores clave a las figuras relevantes para la gestión del área en diferentes esferas de acción: social, económica, político-institucional y académica. Estos fueron reconocidos durante la etapa de diagnóstico, a partir de información secundaria y de los relatos de los entrevistados.

El diagnóstico integrado se elaboró a partir del relevamiento de información secundaria (informes: 11, reportes: 2, libros: 2, archivos de prensa: 2, censos nacionales: 1 , artículos publicados en revistas: 3, tesis de posgrado: 2); la generación de información primaria a partir de entrevistas semi-estructuradas a diferentes actores vinculados a la zona (actores gubernamentales: 1 , asociaciones vecinales: 4 , empresarios inmobiliarios: 4, academia: 2) y dos recorridas de reconocimiento en terreno. Las entrevistas fueron realizadas entre mayo y octubre de 2015 con el objetivo de conocer los principales asuntos de relevancia en el área según la percepción de cada actor social. Previo a las entrevistas se elaboraron preguntas guía con temáticas amplias que era de interés abordar con cada entrevistado. Estas temáticas fueron definidas a partir del relevamiento previo de información secundaria sobre el sitio. El carácter semi-estructurado de las entrevistas permitió que se estableciera un diálogo en el que, además de los temas de interés para el grupo de trabajo, se desarrollaran nuevos aspectos incorporados por cada entrevistado según sus intereses (Valles, 1997). El intercambio fue grabado con el consentimiento del entrevistado y fue transcripto; se tomó

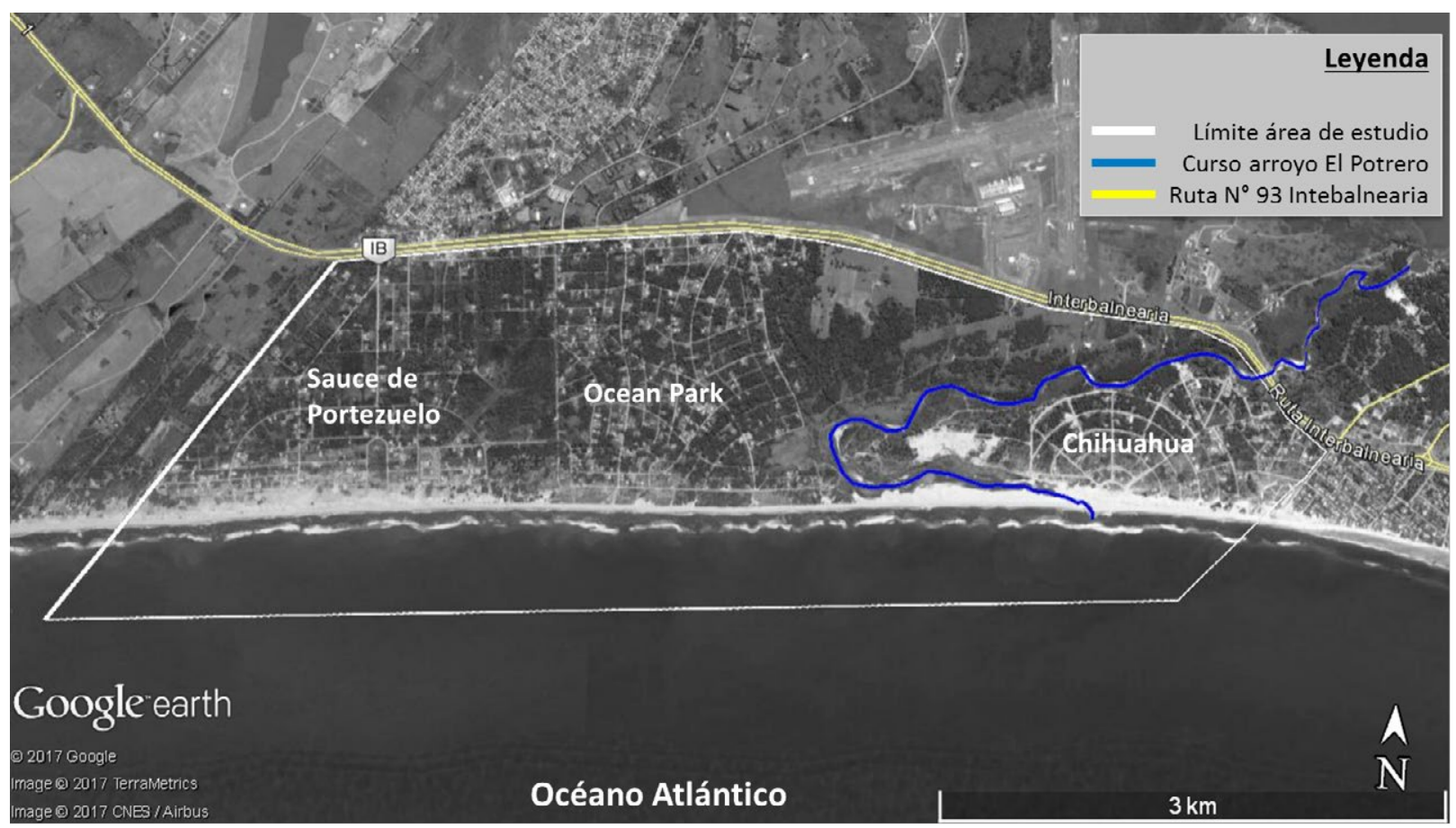

Figura 2. Área de estudio; se muestran los balnearios costeros que la conforman, el curso del arroyo El Potrero y la ruta Interbalnearia $\mathrm{N}^{\circ}$ 93. Fuente: elaboración propia en base a imagen de Google Earth de 24/11/2016. 
nota de lenguaje no verbal observado (gestos de aprobación o desaprobación, tono de voz, etcétera). La información vertida en la entrevista fue utilizada para la reconstrucción de la historia de la zona, la interpretación de las relaciones entre los actores y el conocimiento de los conflictos, problemáticas y oportunidades desde la perspectiva de cada actor.

Se identificaron presiones (conflictos y problemáticas) y oportunidades que fueron la base para la elaboración de propuestas de manejo. Se consideran conflictos a aquellas discrepancias de intereses en torno a la apropiación de los recursos naturales que son declarados por los actores en disputa y adquieren así relevancia pública (Walter, 2009). Se consideran problemáticas a aquellas situaciones de apropiación de los recursos naturales reconocidas como perjudiciales para un determinado actor o como presiones sobre el sistema socioecológico, pero donde los actores involucrados no se enfrentan en carácter de conflicto.

La identificación de conflictos, problemáticas y oportunidades se realizó en base al conocimiento interdisciplinar del equipo de trabajo, recorridas a la zona, revisión documental, entrevistas semi-estructuradas con actores clave y el taller con actores clave.

El procesamiento en talleres internos del grupo de trabajo implicó la descripción de las temáticas, su agrupación geográfica y asociación por similitud. Esta selección fue puesta luego a consideración de un grupo más amplio de actores mediante un taller abierto. Así, la elección de los asuntos se basó principalmente en la visión y percepción de vecinos, agentes inmobiliarios y turísticos, autoridades departamentales, académicos con investigaciones en la zona y el intercambio de conocimiento disciplinar de los integrantes del equipo (Tabla 1).

La información relevada y las conclusiones extraídas a partir de esta etapa fueron puestas a consideración de los actores locales en un espacio de taller abierto de gran relevancia para el proceso de trabajo de este proyecto. El taller se desarrolló el día 24/10/2015 en el Arboretum Lussich. Concurrieron 23 personas: 16 en representación de comisiones vecinales y sociedad civil en general y 7 en representación de instituciones del tercer nivel de gobierno y la academia. El taller se desarrolló en dos etapas, en una primera etapa se realizó una ronda de presentación de los participantes y luego se trabajó en tres subgrupos para identificar temas de interés o conflicto en diferentes subzonas del área de estudio: el arroyo y sus márgenes, la zona costera y la trama urbana. Se utilizaron papelógrafos para plasmar el intercambio y organizar los aportes de los participantes y se llevó a cabo un registro fotográfico, auditivo y escrito de toda la actividad. Finalmente, se estableció un espacio de síntesis y reflexión con todos los presentes.

\section{2) Preparación de programas y proyectos: Plan integrado de manejo}

Finalmente, la generación de propuestas de manejo partió de una primera etapa de identificación de objetivos vinculados a los asuntos de manejo anteriormente seleccionados. Estos objetivos fueron identificados por el equipo de trabajo a partir de los elementos brindados por los actores locales en el taller abierto. Cada uno de los asuntos de manejo dio origen a un programa del cual se desprenden proyectos con acciones específicas. A su vez, todos los programas se agrupan en un plan integrado de manejo denominado «Plan integrado de manejo de la cuenca baja del arroyo El Potrero» (Tabla 2). La estructuración en forma de plan permite reconocer las interacciones entre los asuntos, brindando una mirada holística. Además, se seleccionaron acciones transversales que contribuyen a la implementación de las demás.

\section{Resultados}

\section{Diagnóstico integrado del área}

El turismo se reconoce como la actividad que impulsó los procesos de transformación territorial y sociodemográficos de la zona. A partir del origen y posicionamiento de Punta del Este y Piriápolis, entre 1860 y 1930, como los principales balnearios del departamento, el atractivo turístico de la zona y el flujo de visitantes asociado se intensificó (Díaz Pellicer, 2004). Entre 1930 y 1985 surgieron las urbanizaciones de Punta Ballena, Portezuelo, Sauce de Portezuelo y luego Club del Lago y Ocean Park, sobre las que se centra este trabajo, con una fuerte influencia de arquitectos reconocidos, como el Arq. Antonio Bonet, cuyas obras en el sitio han recibido reconocimiento nacional (Uruguay, 2009). Actualmente las urbanizaciones mantienen características de balneario, con un importante crecimiento de la población en temporada alta (ITU-IDM, 2009; INE, 2014). La naturalidad que estos balnearios han conservado, frente al elevado grado de urbanización de las ciudades turísticas cercanas, ha representado históricamente un atractivo particular (ITU-IDM, 2009).

El paisaje se estructura a través de la interfase entre el arroyo, de una extensión de 6,45 km entre la Laguna del Sauce (declarada Paisaje Protegido en 1989 por el Decreto 367/989) (Uruguay, 1989) y el Océano Atlántico. Se caracteriza por la presencia de parches de bosque ribereño (Alonso Paz y Bassagoda, 2002), un ecosistema lacunar en su desembocadura con barra arenosa, y vestigios de matorral espinoso psamófilo en Sauce de Portezuelo y algunos ejemplares aislados en los demás balnearios (Alonso Paz, 2005). Este paisaje se complementa con extensas superficies de territorio forestadas con las especies exóticas Pinus marítimo, Eucalyptus spp. y Acacia longifolia, ubicadas tanto sobre las dunas transversales como en los márgenes del arroyo (Scasso y Muniz, 1997). Otro elemento de referencia paisajística y cultural es el Arboretum Lussich, desarrollado en el período de creación de Punta del Este y Piriápolis, protegido por la Ley 13.181 (Uruguay, 1963) y declarado Monumento Histórico Nacional en 1984 por la Resolución 1238/984 (Uruguay, 1984).

A nivel faunístico se destaca la presencia de vertebrados marinos de atractivo turístico, como lobos marinos (Arctocephalus australis) y franciscanas (Pontoporia blainvillei), y se observan eventualmente orcas (Orcinus orca) y ballenas Franca Austral (Eubalaena australis) (Iriarte, 2006). Estas especies utilizan la zona como parte de su ruta migratoria y/o área de alimentación. Existen también registros de reptiles marinos como la tortuga verde (Chelonia mydas) y la tortuga cabezona (Caretta caretta) (Defeo, et al., 2009).

Administrativamente la zona se encuentra bajo jurisdicción de dos municipios: Municipio de Piriápolis, al oeste del arroyo, y Municipio de Maldonado, al este del arroyo, lo que representa una complejidad adicional al momento de la aplicación de las medidas de manejo. 


\section{Actores e instituciones}

Los principales actores con injerencia directa en la zona de estudio se presentan en la Tabla 1. No fueron considerados en este trabajo los turistas, actores con presencia masiva pero esporádica en el área. La falta de comunicación e involucramiento de estos actores podría representar una limitante para el éxito de las propuestas de manejo. Tampoco se estableció una consulta directa a actores vinculados al gobierno nacional, aunque su perspectiva fue conocida en forma indirecta a partir de las decisiones adoptadas y del relato de los actores locales.

\section{Presiones y oportunidades}

Tanto los conflictos como las problemáticas identificadas constituyen presiones sobre el sistema socioecológico en estudio, y son forzantes que generan o pueden generar impactos negativos sobre el sistema.

\section{Presiones: conflictos}

Se identificaron cuatro principales conflictos. El primero fue la circulación de vehículos sobre cordón dunar y playa. Pese

\begin{tabular}{|c|c|c|c|}
\hline \multirow{2}{*}{ SECTOR } & \multirow{2}{*}{ ACTOR } & \multicolumn{2}{|c|}{ INSTANCIA DE PARTICIPACIÓN } \\
\hline & & Entrevista & Taller \\
\hline \multirow{3}{*}{$\begin{array}{l}\text { Sociedad civil } \\
\text { organizada }\end{array}$} & Unión Vecinal de Punta Ballena y Lagunas del Sauce y del Diario & SÍ (1 persona) & Sí (3 personas) \\
\hline & Asociación de vecinos de Ocean Park & SÍ (1 persona) & SÍ (1 persona) \\
\hline & Liga de Fomento de Sauce de Portezuelo & Sí (2 personas) & Sí (2 personas) \\
\hline $\begin{array}{l}\text { Sociedad civil } \\
\text { no organizada }\end{array}$ & Vecinos de los diferentes balnearios & NO & SÍ (5 personas) \\
\hline \multirow{2}{*}{$\begin{array}{l}\text { Gobierno } \\
\text { municipal }\end{array}$} & Municipio de Piriápolis & NO & Sí (1 persona) \\
\hline & Municipio de Maldonado & NO & SÍ (1 persona) \\
\hline \multirow{4}{*}{$\begin{array}{c}\text { Gobierno } \\
\text { departamental }\end{array}$} & Dirección de Medio Ambiente (IDM) & SÍ (1 persona) & NO \\
\hline & Dirección de Espacios Públicos (IDM) & NO & NO \\
\hline & Unidad de Gestión Costera (IDM) & NO & NO \\
\hline & Guardavidas & NO & SI (1 persona) \\
\hline \multirow{7}{*}{$\begin{array}{c}\text { Gobierno } \\
\text { nacional }\end{array}$} & Ministerio de Defensa Nacional (MDN) & NO & NO \\
\hline & $\begin{array}{c}\text { Ministerio de Vivienda, Ordenamiento Territorial y Medio } \\
\text { Ambiente (MVOTMA) }\end{array}$ & NO & NO \\
\hline & Ministerio de Transporte y Obras Públicas (MTOP) & NO & NO \\
\hline & Ministerio de Ganadería, Agricultura y Pesca (MGAP) & NO & NO \\
\hline & Ministerio de Turismo (MINTUR) & NO & NO \\
\hline & Ministerio de Energía y Minería (MIEM) & NO & NO \\
\hline & Obras Sanitarias del Estado (OSE) & NO & NO \\
\hline \multirow{2}{*}{ Academia } & Centro Universitario Regional del Este (CURE) & SI (2 personas) & NO \\
\hline & Universidad del Trabajo del Uruguay (UTU) & NO & NO \\
\hline \multirow{7}{*}{ Empresarial } & Hotel Undarius & NO & NO \\
\hline & Hotel El Refugio & NO & SI (2 personas) \\
\hline & Complejo Tío Tom & NO & NO \\
\hline & Dalarna Sma Hotel & NO & SI (1 persona) \\
\hline & Chihuahua Club S.A & NO & NO \\
\hline & Inmobiliaria Punta Ballena & SI (2 personas) & SI (1 persona) \\
\hline & Inmobiliaria Ocean Park & SI (2 personas) & NO \\
\hline
\end{tabular}

Tabla 1. Actores e instituciones reconocidos a partir del diagnóstico, agrupados por sector al que pertenecen e instancias de consulta en las que participaron. 
a la prohibición de la circulación según el Art. 452 de la Ley 16.736 (Uruguay, 1996), se observó que circulan vehículos motorizados, cuatriciclos y motos cross, por ejemplo, en zonas con ecosistemas valiosos y vulnerables, generando procesos erosivos y afectando a las poblaciones de organismos que habitan estos espacios (De Álava, 2008). El conflicto se expresó entre la población residente, turistas, la Dirección Nacional de Medio Ambiente (DINAMA) y la Prefectura Nacional Naval.

El segundo conflicto registrado fue la extracción industrial de áridos en la margen este del arroyo El Potrero. Este sitio se ubica en una zona caracterizada por la normativa departamental como suelo urbano e implica una intervención del medio biofísico que genera una transformación en el paisaje, una alteración en la dinámica de sedimentos, pérdida de vegetación nativa, deterioro de la calidad de agua y efectos negativos sobre la urbanización, como la contaminación sonora, aérea y el deterioro de caminería (los cuales afectan la calidad de vida y el valor inmobiliario de la zona). El conflicto se expresa entre pobladores, sociedades anónimas promotoras de los emprendimientos y la DINAMA.

El tercer conflicto identificado fue la instalación de la Marina de Punta del Este. Este emprendimiento se encuentra en etapa de proyecto desde 1992 en la desembocadura del arroyo (zona de gran importancia ecosistémica y de gran valor paisajístico). Plantea la construcción de un puerto de embarcaciones deportivas y de placer con una urbanización completa (Colacce, et al. 1998). De acuerdo a sus características de megaemprendimiento generaría grandes impactos a nivel biofísico y socioeconómico. El conflicto involucra a pobladores residentes, organizaciones sociales, inversionistas, el gobierno departamental y el gobierno nacional.

Finalmente, el cuarto y último conflicto observado fue la tala ilegal de árboles en la zona. En los tres balnearios del área de estudio se talan árboles de forma ilegal en terrenos baldíos o en las márgenes del arroyo. Esto genera procesos erosivos en los balnearios, degradación de hábitat y alto riesgo de incendios, ya que los restos de podas no son llevados a los puntos verdes dispuestos para ello. El conflicto se expresa entre pobladores, las personas que talan ilegalmente y los municipios encargados de la gestión de residuos verdes.

\section{Presiones: problemáticas}

Las problemáticas observadas estuvieron asociadas en términos generales a una falta de fiscalización y control por parte de las instituciones competentes (cinco casos), a una ausencia de planificación del territorio (cuatro casos), a la necesidad de infraestructura y servicios de los balnearios (cuatro casos) y a la ausencia de iniciativas de gestión conjunta entre la sociedad civil y los municipios (tres casos). Algunas de las problemáticas podrían incluirse en más de una categoría a la vez. A continuación se describen brevemente las diferentes problemáticas. Se ordenan según las categorías que fueron mencionadas anteriormente, sin que eso implique una valoración de importancia.

En el grupo que refiere a la falta de fiscalización y control se evidenció una falta de fiscalización y la necesidad de un mayor control en la zona para atender a actividades ilícitas de distinta índole, desde construcciones a remoción de arena o circulación de vehículos en la playa. Los procesos de respuesta por parte de los órganos competentes son lentos en algunos casos y no se observa una asignación de recursos suficiente para la fiscalización. A su vez, no es efi- ciente el proceso de comunicación de la normativa entre el gobierno y la población. También dentro de este grupo de problemáticas se observó un incremento de construcciones sobre el cordón dunar, dado el proceso de consolidación de los fraccionamientos balnearios. Se destacaron algunas obras edilicias construidas sobre el cordón dunar en el límite de la franja costera pública y otras ubicadas dentro de esa franja, en situación de ilegalidad. Algunas de estas construcciones se encuentran abandonadas. También dentro de esta categoría se constató en algunos de los balnearios la extracción de arena ilegal por parte de particulares (transportada en carros o camionetas) en los médanos ubicados en la faja de 150 metros sobre la línea de la ribera, caracterizada como espacio público. Se advirtió, por otro lado, la colocación de redes de pesca de enmalle en el curso del arroyo, las cuales se encuentran prohibidas de acuerdo a las normas establecidas por la Dirección Nacional de Recursos Acuáticos (DINARA) según resolución del MGAP s/n de noviembre de 2008 (Uruguay, 2008) como medida de conservación en ríos y arroyos del país. Existen terrenos en conflicto dominial de los cuales la administración departamental desconoce la titularidad que poseen, lo cual favorece el desarrollo de actividades ilegales en ellos, como la tala de árboles, ocupaciones, deposición de residuos que forman basurales o la ocupación informal, temporal o permanente de los terrenos, a la vez que no se recaudan tributos por su concepto.

La ausencia de planificación del territorio incluye el acceso peatonal a la paya, que en todos los balnearios ocurre de forma irregular, sin señalización ni infraestructura adecuada, generando una mayor degradación del cordón dunar. Asimismo, se observó que se han establecido estacionamientos informales en accesos a la playa. La falta de planificación de estacionamientos y, por ende, las entradas a la playa de manera desordenada, afecta a los vecinos de la zona y a la vez genera erosión y pérdida de vegetación nativa sobre el cordón dunar. También dentro de esta categoría fue mencionado y pudo constatarse en campo el relleno y desecación del humedal en Ocean Park: con la proyección de ser secado para fines urbanísticos por parte de su propietario, este ecosistema hidrófilo se encuentra en riesgo. Es un hábitat de gran importancia para la alimentación y reproducción de aves acuáticas residentes y migratorias, y funciona como regulador de la dinámica hidrológica de la zona, reduciendo el riesgo de inundaciones. Se mencionó también la preocupación por la ocurrencia de acampadas informales en los márgenes del arroyo. La apropiación temporal y el uso sin control de los espacios en cercanías del arroyo en distintas épocas del año (especialmente en la semana de turismo) generan un aumento de residuos en las márgenes y degradación del hábitat, así como alteraciones en la dinámica sociocultural de los balnearios.

En la categoría de necesidad de infraestructura y servicios se constató una gestión de residuos sólidos insuficiente en playas. Las playas no tienen suficiente infraestructura para la deposición de residuos sólidos y carecen de señalética específica. A su vez, la limpieza de playas ocurre únicamente en los meses de temporada alta, y no es constante a lo largo del año, por lo que se advierte una importante presencia de residuos sólidos provenientes del mar o de las urbanizaciones cercanas. Además, a nivel urbano no existe un sistema de gestión de residuos sólidos adecuado. Se observa una insuficiente cantidad de recipientes y ausencia de señalización o cartelería que permita una orientación o sensibilización en cuanto al tema. Tampoco se aprecia una clasificación efectiva 
de residuos que permita su ingreso al sistema de reutilización o clasificación. Se generan basurales en terrenos baldíos, ocurre transporte de residuos por efecto de la escorrentía y la deposición de podas en lugares no habilitados para tal fin. Esto genera contaminación y aumenta el riesgo de incendios, afectando directamente la calidad de vida de los pobladores. También dentro de esta categoría se reconoce que el sistema de pluviales en las zonas urbanizadas está incompleto. Ha sido desarrollado de forma incipiente en algunas zonas pero es inexistente en la mayoría de los balnearios, lo que genera procesos erosivos en el entramado de caminería y en algunos terrenos y espacios públicos, así como la inundación de los balnearios cuando las precipitaciones son copiosas. Finalmente, no existe sistema de saneamiento en ninguno de los balnearios del área de estudio. Esto genera contaminación por efluentes sanitarios en la trama urbana y en la zona de playa, situación que se agrava en algunas zonas sin acceso a agua potable que utilizan agua subterránea. Esta situación empeora por un incipiente sistema de pluviales que no evita inundaciones.

Por último, dentro de la categoría de ausencia de iniciativas de gestión conjunta entre la sociedad civil y los municipios se reconoció la ausencia de coordinación en la apertura de la represa de Laguna del Sauce. La apertura ocasional de las reclusas de la represa, ubicada aguas arriba en el arroyo, es estimada según los pobladores como generadora de importantes disturbios en su hidrodinámica, aportando altas concentraciones de nutrientes (debido al estado eutrófico actual de la laguna) e incluso le adjudican inundaciones que afectan los predios (urbanos y suburbanos) ubicados sobre la planicie de inundación del arroyo. Se observó también que los espacios públicos se encuentran parcial o totalmente abandonados, en riesgo de ser ocupados, con presencia de vegetación alta, falta de caminería, que impide el acceso, y, en algunos casos, se transforman en vertederos de residuos sólidos, con el consecuente riesgo sanitario. Actualmente se encuentran invisibilizados y/o subutilizados sin gestión institucional y las asociaciones vecinales mencionan su interés por revalorizarlos y acondicionarlos. Se constató también que existen infraestructuras en estado de abandono o semi-abandono que podrían ser reutilizadas o revalorizadas. Un ejemplo es el mirador ubicado en Sauce de Portezuelo, de alto valor patrimonial y cultural para la zona. Otro ejemplo lo constituye el hotel en situación de semi-abandono ubicado también en Sauce de Portezuelo, al cual están asociadas piletas de potabilización de agua salada ubicadas en la playa, también en desuso.

\section{Oportunidades}

Las oportunidades relevadas se encontraron asociadas a la creación de espacios de articulación y gestión conjunta entre los miembros de la sociedad civil, la puesta en valor del paisaje y la posibilidad de trabajar en el ordenamiento de la trama balnearia dada su moderada consolidación.

Las asociaciones vecinales, que alcanzaron un total de tres, se encontraron en estados diferentes de consolidación, por lo que la articulación entre ellas representa una muy buena oportunidad de desarrollo con aportes mutuos. Hay además un fuerte interés por parte de los pobladores residentes de abordar las problemáticas y conflictos de la zona, lo cual, sumado a que varios de esos conflictos y problemáticas son compartidos por todos los balnearios del área de estudio, crea una ventana de oportunidad para generar espacios de participación y articulación entre las asociaciones vecinales.

Otra oportunidad reconocida fue el potencial uso turístico y recreativo del arroyo. El arroyo y sus márgenes constituyen un ecosistema caracterizado por destacados atributos naturales y una importante valoración socio-cultural por parte de los pobladores locales y los turistas. Esto constituye una oportunidad para trabajar hacia la puesta en valor de este ecosistema, promoviendo actividades turísticas y recreativas que realcen su valor paisajístico, sociocultural y patrimonial, desestimulando y restringiendo actividades que lo deterioren.

Finalmente, se reconoció que el hecho de que los balnearios del área de estudio aún no se encuentren completamente consolidados genera la oportunidad de replantear el modelo territorial de la trama urbana, disminuyendo el impacto que esta pueda tener sobre la matriz biofísica en la que se desarrolla.

\section{Selección de asuntos de manejo}

El resultado de este análisis fue la selección de cuatro asuntos clave de manejo: i) fortalecimiento de la gobernanza; ii) aumento de la integridad ambiental de la costa balnearia; iii) conservación del arroyo y sus márgenes, y iv) consolidación de una trama urbana de bajo impacto ambiental. En cada uno de estos asuntos están incluidos los conflictos, problemáticas y oportunidades reconocidas (Tabla 2).

\section{Propuestas de manejo}

Se generó un «Plan integrado de manejo de la cuenca baja del arroyo El Potrero» (Tabla 2) incluyendo los programas y los proyectos. La estructuración en forma de plan permite reconocer las interacciones entre los asuntos, brindando una mirada holística. Como acciones transversales se identificaron la necesidad de contar con una estrategia de comunicación y difusión de las propuestas y de otras informaciones que sean de interés para la zona o sean generadas desde ella, y la elaboración de una línea de base del área de estudio que sustentará las propuestas posteriores y será la referencia para el monitoreo de las acciones.

\section{Discusión}

En este trabajo se obtuvo un diagnóstico de la cuenca baja del arroyo El Potrero mediante la síntesis de información que se encontraba dispersa en diferentes fuentes. El espacio de interacción propuesto entre diversos actores permitió además lograr acuerdos en torno a los asuntos de manejo prioritarios para la zona para posteriormente generar las propuestas de manejo. El intercambio entre diversos actores económicos, sociales y políticos, en el que se ponen en diálogo las experiencias y conocimientos de cada actor, fue fundamental para evidenciar la complejidad de los sistemas socioecológicos y aplicar diferentes herramientas y enfoques para gestionarlos como también ha ocurrido en otros ámbitos de aplicación del MCI (Olsen y Ochoa, 2007). Para cada uno de los programas de manejo elaborados se discute a continuación la descripción de la trama socioecológica que se interpretó en función de las presiones y oportunidades relevadas. 


\begin{tabular}{|c|c|c|c|c|}
\hline \multicolumn{5}{|c|}{ PLAN INTEGRADO DE MANEJO DE LA CUENCA BAJA DEL ARROYO EL POTRERO } \\
\hline \multicolumn{5}{|c|}{ Actividad transversal: Elaboración línea de base para el área } \\
\hline \multicolumn{5}{|c|}{ Actividad transversal: Estrategia de comunicación y difusión transversal } \\
\hline Programas & Proyectos & Objetivos & $\begin{array}{c}\text { Problemáticas }(\mathrm{P}) \\
\text { Conflictos }(\mathrm{C}) \\
\text { Oportunidades }(\mathrm{O}) \\
\end{array}$ & Actores clave \\
\hline \multirow{2}{*}{$\begin{array}{l}\text { Fortalecimiento } \\
\text { de la gobernanza } \\
\text { a través de la } \\
\text { sociedad civil } \\
\text { organizada }\end{array}$} & $\begin{array}{l}\text { Formación de } \\
\text { actores locales y } \\
\text { fortalecimiento } \\
\text { de los espacios } \\
\text { de gobernanza }\end{array}$ & $\begin{array}{l}\text { Generar espacios de encuentro } \\
\text { y construcción colectiva que } \\
\text { potencien el desarrollo de redes } \\
\text { interorganizacionales y con } \\
\text { el gobierno departamental y } \\
\text { municipal. }\end{array}$ & \multirow{2}{*}{$\begin{array}{l}\text { - Falta de fiscalización }(\mathrm{P}) \\
\text { - Articulación entre } \\
\text { asociaciones vecinales de la } \\
\text { zona }(\mathrm{O})\end{array}$} & \multirow{2}{*}{$\begin{array}{l}\text { Organizaciones vecinales } \\
\text { de Ocean Park, Sauce } \\
\text { de Portezuelo y Punta } \\
\text { Ballena, Intendencia de } \\
\text { Maldonado, Municipio } \\
\text { de Maldonado, Muni- } \\
\text { cipio de Piriápolis, Pre- } \\
\text { fectura Nacional, CURE } \\
\text { Maldonado (Udelar) }\end{array}$} \\
\hline & $\begin{array}{l}\text { Observatorio } \\
\text { Ambiental } \\
\text { Comunitario }\end{array}$ & $\begin{array}{l}\text { Generar un espacio de } \\
\text { coordinación permanente de la } \\
\text { sociedad civil organizada y no } \\
\text { organizada desde el cual se genere } \\
\text { información para el monitoreo y } \\
\text { control ambiental de la zona. }\end{array}$ & & \\
\hline \multirow{3}{*}{$\begin{array}{l}\text { Integridad } \\
\text { ambiental de la } \\
\text { costa balnearia }\end{array}$} & $\begin{array}{l}\text { Vegetación } \\
\text { costera }\end{array}$ & $\begin{array}{l}\text { Conservar y dar comienzo a la } \\
\text { restauración de la vegetación } \\
\text { nativa presente en la franja } \\
\text { costera comprendida por el } \\
\text { espacio público de la costa. }\end{array}$ & \multirow{3}{*}{$\begin{array}{l}\text { - Circulación de vehículos } \\
\text { sobre cordón dunar y playa } \\
\text { (C) } \\
\text { - Construcción sobre el } \\
\text { cordón dunar }(\mathrm{P}) \\
\text { - Extracción ilegal de arena } \\
\text { en zona de playa }(\mathrm{P}) \\
\text { - Accesos a la playa } \\
\text { irregulares (P) } \\
\text { - Ausencia de sistema de } \\
\text { gestión de residuos sólidos en } \\
\text { playas (P) } \\
\text { - Establecimiento de } \\
\text { estacionamientos informales } \\
\text { en accesos a playa (P) }\end{array}$} & \multirow{3}{*}{$\begin{array}{l}\text { Organizaciones vecinales } \\
\text { de Ocean Park, Sauce } \\
\text { de Portezuelo y Punta } \\
\text { Ballena, establecimientos } \\
\text { turísticos de la zona, } \\
\text { MVOTMA, Municipio } \\
\text { de Piriápolis, Municipio } \\
\text { de Maldonado e } \\
\text { Intendencia de } \\
\text { Maldonado (Dirección } \\
\text { de Medio Ambiente), } \\
\text { CURE Maldonado } \\
\text { (Udelar). }\end{array}$} \\
\hline & $\begin{array}{l}\text { Huellas en la } \\
\text { costa }\end{array}$ & $\begin{array}{l}\text { Regular los usos que ocurren } \\
\text { en la costa de forma de mitigar } \\
\text { el impacto de las actividades } \\
\text { antrópicas en el ambiente costero. }\end{array}$ & & \\
\hline & $\begin{array}{l}\text { Parque costero } \\
\text { balneario }\end{array}$ & $\begin{array}{l}\text { Establecer un parque costero } \\
\text { balneario que regule las } \\
\text { actividades que ocurren en el } \\
\text { espacio costero, revalorizando } \\
\text { el paisaje y la importancia del } \\
\text { sistema dunar y promoviendo un } \\
\text { turismo sustentable. }\end{array}$ & & \\
\hline \multirow[b]{2}{*}{$\begin{array}{l}\text { Conservación } \\
\text { del arroyo y sus } \\
\text { márgenes }\end{array}$} & $\begin{array}{l}\text { El arroyo y sus } \\
\text { servicios socio- } \\
\text { ecosistémicos }\end{array}$ & $\begin{array}{l}\text { Identificar y poner en valor los } \\
\text { servicios ecosistémicos brindados } \\
\text { por el arroyo y proponer un plan } \\
\text { de desarrollo turístico de bajo } \\
\text { impacto ambiental que promueva } \\
\text { su conservación y restauración. }\end{array}$ & \multirow{2}{*}{$\begin{array}{l}\text { - Extracción industrial de } \\
\text { áridos en la margen este del } \\
\text { arroyo El Potrero (C). } \\
\text { - Instalación de Marina Punta } \\
\text { del Este Chihuahua Club S.A } \\
\text { (C). } \\
\text { - Relleno y desecación del } \\
\text { humedal en Ocean Park (P). } \\
\text { - Acampadas informales en } \\
\text { los márgenes del arroyo (P). } \\
\text { - Colocación ilegal de redes } \\
\text { de pesca en el curso del } \\
\text { arroyo (P). } \\
\text { - Apertura de la represa de } \\
\text { Laguna del Sauce (P). } \\
\text { - Uso turístico y recreativo } \\
\text { del arroyo (O). }\end{array}$} & \multirow{2}{*}{$\begin{array}{l}\text { Organizaciones vecinales } \\
\text { de Sauce de Portezuelo, } \\
\text { Ocean Park y Punta } \\
\text { Ballena, establecimientos } \\
\text { turísticos de la } \\
\text { zona, APROTUR, } \\
\text { MVOTMA, OSE, MIEM } \\
\text { (DINAMIGE), UdelaR } \\
\text { (CURE Maldonado), } \\
\text { Comisión de Cuenca } \\
\text { de la Laguna del } \\
\text { Sauce, Prefectura } \\
\text { Nacional, Municipio de } \\
\text { Piriápolis, Municipio de } \\
\text { Maldonado, Intendencia } \\
\text { de Maldonado. }\end{array}$} \\
\hline & $\begin{array}{l}\text { Plan parcial de } \\
\text { la cuenca baja } \\
\text { del arroyo }\end{array}$ & $\begin{array}{l}\text { Integrar la cuenca del arroyo } \\
\text { El Potrero a un Plan Parcial } \\
\text { de Ordenamiento Territorial } \\
\text { con objetivos y acciones que } \\
\text { apunten a promover su integridad } \\
\text { ecosistémica. }\end{array}$ & & \\
\hline \multirow[b]{2}{*}{$\begin{array}{l}\text { Desarrollo de una } \\
\text { trama urbana } \\
\text { de bajo impacto } \\
\text { ambiental }\end{array}$} & $\begin{array}{l}\text { Hacia un } \\
\text { sistema de } \\
\text { saneamiento y } \\
\text { de pluviales en } \\
\text { los balnearios }\end{array}$ & $\begin{array}{l}\text { Impulsar acciones hacia un } \\
\text { sistema de saneamiento que } \\
\text { considere la participación de la } \\
\text { población desde la gestión, el } \\
\text { diseño e implementación. }\end{array}$ & \multirow{2}{*}{$\begin{array}{l}\text { - Tala ilegal de árboles en zona } \\
\text { urbana }(\mathrm{C}) \text {. } \\
\text { - Terrenos en conflicto } \\
\text { dominial }(\mathrm{P}) \text {. } \\
\text { - Ausencia de un sistema de } \\
\text { gestión de residuos sólidos }(\mathrm{P}) \text {. } \\
\text { - Sistema de pluviales } \\
\text { incompleto }(\mathrm{P}) \text {. } \\
\text { - Ausencia de sistema de } \\
\text { saneamiento }(\mathrm{P}) \text {. } \\
\text { - Espacios públicos } \\
\text { invisibilizados y/o } \\
\text { subutilizados }(\mathrm{P}) . \\
\text { - Infraestructuras } \\
\text { abandonadas }(\mathrm{P}) \text {. } \\
\text { - Consolidación moderada de } \\
\text { los balnearios }(\mathrm{O}) \text {. }\end{array}$} & \multirow[b]{2}{*}{$\begin{array}{l}\text { MVOTMA (DINOT), } \\
\text { Organizaciones vecinales } \\
\text { de Ocean Park, Sauce } \\
\text { de Portezuelo y Punta } \\
\text { Ballena, Municipio de } \\
\text { Piriápolis, Municipio } \\
\text { de Maldonado, } \\
\text { MVOTMA, Intendencia } \\
\text { de Maldonado, CURE } \\
\text { Maldonado (UdelaR). }\end{array}$} \\
\hline & $\begin{array}{l}\text { Revalorización } \\
\text { de la trama } \\
\text { urbana de los } \\
\text { balnearios }\end{array}$ & $\begin{array}{l}\text { Promover una planificación } \\
\text { participativa de desarrollo de } \\
\text { los balnearios para: revalorizar } \\
\text { los espacios públicos de la trama } \\
\text { urbana, revalorizar y recuperar } \\
\text { espacios naturales, reutilizar } \\
\text { infraestructuras abandonadas } \\
\text { y regularizar los terrenos en } \\
\text { conflicto dominial. }\end{array}$ & & \\
\hline
\end{tabular}

Tabla 2. Programas y proyectos que componen el «Plan integrado de manejo de la cuenca baja del arroyo El Potrero» y sus principales objetivos. Se detallan a su vez los conflictos, problemáticas y oportunidades involucrados en cada programa y los actores que es importante que se involucren. 


\section{Fortalecimiento de la gobernanza a través de la sociedad civil organizada}

Se constató la existencia de agrupaciones vecinales (Unión vecinal de Punta Ballena y Lagunas del Sauce y del Diario, Asociación de vecinos de Ocean Park, Liga de Fomento de Sauce de Portezuelo, entre otras en formación) con diferente nivel de incidencia en la generación de políticas públicas y diferente grado de consolidación interna. El nivel de incidencia se interpretó a partir de los antecedentes de cada agrupación de participación en espacios de interacción con el nivel político u otras agrupaciones o instituciones. En base a esto se propone que los principales aspectos a promover tengan que ver con el desarrollo de capacidades de movilización de recursos, de incidencia política y de diálogo interorganizacional, todo esto enmarcado en un contexto de problemáticas y conflictos ambientales diversos (Ernoul y Wardell-Johnson, 2013).

\section{Integridad ambiental de la costa balnearia}

En la costa balnearia de la zona de estudio se encontraron relativamente preservadas algunas unidades ambientales de importante valor ecosistémico, como dunas y playas. Se observaron, además, parches de vegetación nativa costera como el matorral espinoso psamófilo, lo que le confiere un importante valor ecosistémico al lugar. Los fraccionamientos balnearios presentes en el área aún no se encontraron completamente desarrollados, si bien su proceso de consolidación se ha visto acelerado en los últimos años. Aunque esta fracción de la costa balnearia posee características agrestes y un valor paisajístico altamente reconocido por los turistas y los residentes permanentes, la zona costera de estos balnearios se encuentra sometida a una fuerte presión por el desarrollo inmobiliario y el turismo, por lo que se hace necesario implementar una gestión que asegure su integridad a largo plazo (ITU-IDM, 2009).

\section{Conservación del arroyo y sus márgenes}

El arroyo El Potrero incluye una diversidad de ambientes naturales que sustentan una gran diversidad faunística y florística, que en su conjunto le otorgan un destacado valor ecosistémico y paisajístico (Brazeiro, et al., 2009). En este trabajo se observó la ocurrencia de complejas interacciones entre las actividades humanas y el sistema natural por las diversas actividades, principalmente recreativas (turismo convencional y naturista, travesías en canoa y kayak, senderismo, acampadas) y extractivas (tala de monte y extracción de áridos en las márgenes), que ejercen fuertes presiones sobre el sistema socioecológico. Estas interacciones se observaron en una interfase de espacio público-privado dentro de la trama urbana y los predios suburbanos y rurales linderos al arroyo, y generaron un escenario aún más intrincado para su manejo. La complejidad de procesos e interacciones que sustenta el arroyo como unidad ambiental amerita que sea preservado con el fin de asegurar la sustentabilidad ambiental de la zona (De Álava, 2008).

\section{Desarrollo de una trama urbana de bajo impacto ambiental}

Los balnearios costeros de la zona de estudio han enfrentado un rápido proceso de expansión y consolidación y, si bien existen diferentes grados de consolidación entre ellos, se observa un crecimiento sostenido de la mancha urbana. Los fraccionamientos correspondientes a los balnearios en estudio fueron realizados sin considerar la matriz biofísica sobre la que se establecieron. Si bien su proceso de consolidación se ha visto acelerado, aún es posible gestionarlo y rediseñarlo con el objetivo de reducir el impacto ambiental que genera sobre ecosistemas frágiles como el arroyo y la franja costera. Por otro lado, la relativa baja densidad poblacional de estos balnearios condiciona el acceso a los servicios públicos que toda urbanización necesita. No cuentan con sistema de saneamiento, ni con un correcto sistema de pluviales y gestión de residuos sólidos, todo lo cual genera impactos negativos sobre el ecosistema y disminuye la calidad de vida de los pobladores. La implementación de este programa pretende alcanzar una gestión territorial y ambiental participativa que apunte a disminuir el impacto ambiental derivado de la consolidación urbana y mejorar la calidad de vida de sus habitantes (ITU-IDM, 2009).

\section{Desafíos y aprendizajes en torno a la implementación de un enfoque MCI}

En diferentes partes del mundo, los principales desafíos para la implementación del MCI se encuentran en la falta de financiación y en el surgimiento de conflictos de intereses y pujas de poder (Ernoul y Wardell-Johnson, 2013). Asimismo, las brechas comunicacionales existentes entre las diferentes disciplinas de un equipo interdisciplinario de investigación y los gestores u otros sectores, las políticas y tiempos de los gestores, y las insuficiencias de las estructuras democráticas para incluir las necesidades de los actores locales también son limitantes para la implementación del MCI (Shipman y Stojanovic, 2007). En ese escenario de complejidad es frecuente que los procesos de MCI no se desarrollen en su cabalidad (Ernoul y Wardell-Johnson, 2013), tendiendo a reducir las formas de participación social a su mínima expresión y resultando en procesos que involucran únicamente diferentes esferas del nivel gubernamental y la academia (Shipman y Stojanovic, 2007; CMCI, 2011). A partir de la experiencia aquí presentada puede sostenerse, sin embargo, que en el transcurso del proceso de MCI los actores y sus relaciones se transformaron, por lo que la sola generación de un espacio de intercambio y participación de actores diversos representa un avance hacia el cumplimiento de los objetivos del MCI. El proceso de generación de propuestas de manejo promueve el fortalecimiento de los diversos actores y la generación de capacidades para la toma de decisiones.

El espacio de intercambio propuesto en el marco de este trabajo ha sido útil para generar lazos entre los actores y entablar debates en torno a problemáticas puntuales combinando las capacidades de los presentes, sorteando las dificultades de comunicación y brindando la posibilidad de concretar acciones conjuntas. El rol de este equipo de trabajo ha sido fundamental para la articulación entre los actores, ya que fomentó el contacto entre los miembros de la comunidad a través del taller abierto. Esto representó, a su vez, una limitación en la propuesta, dado que el proceso fue sostenido por la intervención de un grupo externo y no se logró que sea liderado por actores locales.

Este tipo de prácticas tienden a disminuir la brecha entre la producción de conocimiento científico y su utilización por parte de los actores en territorio, así como a generar espacios para la conjugación de ese conocimiento con otras formas de 
saber no académico (Conde, 2013). Este enfoque se vuelve imprescindible cuando se trata de atender situaciones con alto grado de incertidumbre, como son las que refieren a sistemas socioecológicos y particularmente las vinculadas a la zona costera (Allen y Garmestani, 2015). Esto implica, además, establecer un proceso de aprendizaje basado en una revisión continua mediante el monitoreo de avances, resultados y objetivos, en una lógica adaptativa (Armitage, et al., 2009), concepción que es necesario afianzar para posicionarla frente a las lógicas de inmediatez y obtención de resultados que se ha vuelto hegemónica y no es acorde a la dinámica de los procesos socioecológicos.

\section{Reconocimientos}

El presente trabajo se desarrolló en el marco de la Maestría en Manejo Costero Integrado del Cono Sur, CURE, Udelar. El equipo de trabajo estuvo conformado por Juan Manuel Cardozo, Carla Bruzzone, Lucía Bergós y Cecilia Castaño, bajo la tutoría de Laura Brum. Agradecemos especialmente a las asociaciones vecinales, sus representantes y demás actores locales e institucionales que participaron en las diferentes instancias de este trabajo, por su colaboración y motivación.

\section{Referencias}

Armitage, D., Plummer, R., Berkes, F., Arthur, R., Charles, A., Davidson-Hunt, I., Diduck, A., Doubleday, N., Johnson, D., Marschke, M., McConney, P., Pinkerton, E. y Wollenberg, E., 2009. Adaptive co-management for socialecological complexity. En: Frontiers in Ecology and the Environment, (6), pp.95-102.

Aguirre, M., Ávila, S., Collazo, D., Píriz, C. y Varela, C., 2001. Una experiencia de gestión integrada costera. Programa ECOPLATA 1999 - 2001. Montevideo: Programa ECOPLATA. ISBN: 9974-7646-2-9.

Allen, C. y Garmestani, A., 2015. Adaptive management of social-ecological systems. New York: Springer. ISBN: 97894-017-9682-8.

Alonso Paz, E., 2005. Desarrollo urbano-turístico y monte psamófilo. En: Guayubira. Seminario Compartiendo Conocimientos sobre el Monte Indígena. Montevideo, Uruguay (15 de octubre de 2005). Montevideo: Guayubira.

Alonso Paz, E. y Bassagoda, M., 2002. La vegetación costera del SE uruguayo: ambientes y biodiversidad. En: Documentos de Divulgación. Museo Nacional de Historia Natural y Antropología, (5), pp.1-6.

Baliero, W., Biasco, E., Conde, D., Cortazzo, R., Fossati, M., Gorfinkiel, D., Lorenzo, E., Menafra, R., Píriz, C. y Roche, I., 2006. Estudio de Base sobre el Estado del Manejo Costero Integrado en Uruguay: práctica, capacitación e investigación. Proyecto «Sustentabilidad de la Zona Costera Uruguaya» (AUCC-CIDA). Montevideo/Halifax: Universidad de la República/Dalhousie University.

Brazeiro, A., Toranza, C. y Bartesaghi, L., 2009. Proyecto Biodiversidad Costera. Resultado 7 del Proyecto URU 06/016: 2.3.3. Montevideo: EcoPlata-UdelaR/Facultad de Ciencias.

Colacce, C., Alsina, J., Anastasía, L., Colacce, M., Muniz, P., Ross, P. y Scasso, F., 1998. Informe resumen Estudio de Impacto Ambiental Marina Punta del Este. Informe inédito.

Conde, D., 2013. Costas. Montevideo: Comisión del Bicentenario, IMPO. (Colección Nuestro Tiempo, 9)
De Álava, D., 2008. Proyecto de restauración sistema de playas y dunas frontales de la Bahía de Portezuelo [En línea]. Maldonado: Unión Vecinal de Punta Ballena y Lagunas del Sauce y del Diario. [Consulta 31 de marzo de 2017]. Disponible en: http://www.puntaballena.org/macros/ TextContent_M_UV.jsp?contentid=977\&version=1

Defeo, O., Horta. S., Carranza. A., Lercari, D., de Álava, A., Gómez, J., Martínez, G., Lozoya, JP. y Celentano, E., 2009. Hacia un manejo ecosistémico de pesquerías. áreas marinas protegidas en Uruguay. Montevideo: Facultad de Ciencias, DINARA.

Díaz Pellicer, L. 2004. El turismo receptivo en Uruguay (19301986). Montevideo: Unidad Facultad de Ciencias Sociales. (Documento de trabajo, 65).

Ernoul, L. y Wardell-Johnson, A., 2013. Governance in integrated coastal zone management: a social networks analysis of cross-scale collaboration. En: Environmental Conservation, 40, pp.231-240.

Hildebrand, L., 2002. Integrated coastal management: lessons learned and challenges ahead. Citado en: Conde, D., 2013. Costas. Montevideo: Comisión del Bicentenario, Dirección Nacional de Impresiones y Publicaciones Oficiales (IMPO). (Colección Nuestro Tiempo, 9). pp. 30.

GESAMP, Grupo Mixto de Expertos OMI/FAO/UNESCOCOI/OMM/OMS/OIEA/Naciones Unidas/PNUMA sobre los Aspectos Científicos de la Protección del Medio Marino, 1999. La contribución de la ciencia al manejo costero integrado [En línea]. Roma: FAO. (Informes y Estudios, 61). [Consulta 31 de marzo de 2017]. Disponible en: http://www.fao.org/3/contents/dc824e26-b1b7-568d8770-1f9347ecb063/W1639E00.HTM.

Iriarte, V., 2006. Killer whale (Orcinus orca) occurrence at Isla de Lobos, Uruguay. En: Latin American Journal of Aquatic Mammals, 5(1), pp.73-76.

INE, 2014. Uruguay en cifras 2013 [En línea]. Montevideo: Instituto Nacional de Estadística. [Consulta 31 de marzo de 2017]. Disponible en: http://www.ine.gub.uy/uruguayen-cifras

Instituto de Teoría y Urbanismo e Intendencia de Maldonado, 2009.Taller territorial microrregión: Laguna del Sauce, Portezuelo, Punta Ballena, Laguna del Diario [En línea]. [s.l.]: Facultad de Arquitectura, Intendencia de Maldonado. (Cuaderno, 01). [Consulta 31 de marzo de 2017]. Disponible en: http://www.fadu.edu.uy/itu/files/2014/11/01-laguna-delsauce-potezuelo-laguna-del-diario.pdf

MCISur, 2011. Manejo costero integrado en Uruguay: ocho ensayos interdisciplinarios [En línea]. Montevideo: Centro Interdisciplinario para el Manejo Costero Integrado del Cono Sur, UDELAR, CIDA. [Consulta 31 de marzo de 2017]. Disponible en: http://www.unesco.org/new/ fileadmin/MULTIMEDIA/FIELD/Montevideo/pdf/MCIManejoCosteroIntegradoUruguay.pdf

Ochoa, E. y Olsen, S., 2007. El porqué y el cómo de una línea de base para gobernanza en los ecosistemas costeros [En línea]. Guayaquil: [s.n.]. [Consulta 24 de junio de 2017]. Disponible en: http://pdf.usaid.gov/pdf_docs/pnaeb386.pdf

Olsen, S. y Ochoa, E., 2004. Marco metodológico y conceptual para la planeación e implementación del manejo de ecosistemas costeros [En línea]. Rhode Island: Centro de Recursos Costeros de la Universidad de Rhode Island, Fundación EcoCostas, Manejo Costero Integrado. [Consulta 31 de marzo de 2017]. Disponible en: http:// www.ecocostas.org/index.php/glosario. 
Ostrom, E., 2009. A general framework for analyzing sustainability of social-ecological systems. En: Science, (325), pp.419-422.

Scasso, F., Muniz, P. 1997. Informe de impacto ambiental del Proyecto Marina Punta del Este: Medio biológico. Montevideo: Informe inédito.

Shipman, B. y Stojanovic, S., 2007. Facts, fictions and failures of integrated coastal zone management in Europe. En: Coastal Management, 35(2), pp.375-398.

Sorensen, J., 2000. Building a global database of ICM efforts. Boston: University of Massachusetts Press.

United Nations, 1993. Earth summit: Agenda 21, the United Nations Programme of Action from Rio [En línea]. New York: United Nations. [Consulta 31 de marzo de 2017]. Disponible en: https://sustainabledevelopment.un.org/ outcomedocuments/agenda21

Uruguay. Ley 13.181, de 24 de octubre de 1963. Diario Oficial, 7 de noviembre de 1963, No. 16.760, p. 270A
Uruguay. Resolución 1238/984, de 27 de diciembre de 1984. Diario Oficial, 11 de julio de 1985, No. 21.976, p. 112A

Uruguay. Decreto 367/989, de 2 de agosto de 1989. Diario Oficial, 24 de noviembre de 1989, No. 22.993, p. 290A

Uruguay. Ley 16.736, de 5 de enero de 1996. Diario Oficial, 12 de enero de 1996, No. 24.457, p. 774 A

Uruguay. Resolución s/n del MGAP, 5 de noviembre de 2008. Diario Oficial, 19 de noviembre de 2008, No. 27.615, p.735A

Uruguay. Resolución 813/009, de 13 de agosto de 2009. Diario Oficial, 2 de setiembre de 2009, No. 27.806, p. 10B

Valles, M., 1997. Técnicas cualitativas de investigación social: Reflexión metodológica y práctica profesional. Madrid: Síntesis. ISBN: 84-7738-449-5.

Walter, M., 2009. Conflictos ambientales, socioambientales, ecológico distributivos, de contenido ambiental. Reflexionando sobre enfoques y definiciones. En: Boletín ECOS, CIP-Ecosocial, (6), pp.2-9. 